\title{
Síndrome da realimentação: um quadro potencialmente fatal
}

\author{
Refeeding syndrome: a potential fatal condition
}

\author{
Marcia Souza Carvalho', Karina Franco Zihlmann' \\ 'Universidade Federal de São Paulo, Departamento Saúde, Educação e Sociedade, Santos (SP), Brasil.
}

\section{RESUMO}

Objetivo: Realizou-se uma pesquisa com o objetivo de caracterizar os conhecimentos e práticas de uma equipe multiprofissional de saúde de alta complexidade quanto aos quadros de desnutrição e a Síndrome de Realimentação (SR). Além disso, a partir das informações colhidas na pesquisa, pretende-se embasar a construção de um Produto Técnico em Educação (PTE) na forma de um protocolo de avaliação multiprofissional para subsidiar um processo de Educação Permanente (EP) sobre este quadro complexo e fatal. Método: A estratégia metodológica foi qualitativa com uso grupos focais como instrumento e envolvendo 13 profissionais de saúde de um hospital geral da cidade de Santos, na região da Baixada Santista - São Paulo - Brasil. Resultados: Os resultados evidenciaram que o desconhecimento sobre a SR pode ter como consequência negligência na atenção a este quadro complexo. Conclusão: Conclui-se que há necessidade de aprofundar essa temática durante a formação na graduação, bem como propor ações de Educação Permanente nos serviços de saúde de modo a abordar adequadamente as necessidades de cuidado específicas desses pacientes.

PALAVRAS-CHAVE: Síndrome da realimentação. Desnutrição. Terapia nutricional. Grupo focal.

Recebido: Abr. 20, 2021 Aceito: Jun. 24, 2021

\section{COMO CITAR ESTE ARTIGO} Carvalho MS, Zihlmann KF. Síndrome da realimentação: um quadro potencialmente fatal. Interdisciplinary Journal of Health Education. 1-9. https://doi.org/10.4322/ijhe.2020.016

\section{CORRESPONDÊNCIA}

Karina Franco Zihlmann

Universidade Federal de São Paulo, Instituto Saúde e Sociedade, Campus Baixada Santista, Rua Silva Jardim, 136, Vila Mathias, CEP 11015-020, Santos (SP), Brasil

karina.zihlmann@unifesp.br

\section{ABSTRACT}

Objective: A survey was carried out that aimed to characterize the knowledge and practices of a multidisciplinary health team regarding malnutrition and the Refeeding Syndrome (RS). In addition, from the research's collected information, it is intended to support the construction of a Technical Product in Education (TEP) in the form of a multidisciplinary assessment protocol to support a process of Permanent Education (PE) on this complex and fatal syndrome. Methods: The methodological strategy was qualitative, using focus groups instrument and involving 13 health professionals from a general hospital at Santos city, in Baixada Santista region - São Paulo - Brazil. Results: The results showed that the lack of knowledge about RS can result in neglecting proper attention to this syndrome. Conclusion: It is concluded that there is a need to teach this theme during undergraduate training, as well as to propose Permanent Education actions in health services in order to properly address these patient's specific care needs.

KEYWORDS: Refeeding syndrome. Malnutrition. Nutritional therapy. Focus group.

\section{FONTE DE FINANCIAMENTO}

Financiamento próprio

\section{CONFLITO DE INTERESSE}

As autoras referem não haver

nenhum conflito de interesse.

O estudo foi realizado na Universidade Federal de São Paulo, Baixada

Santista, Santos (SP), Brasil. 


\section{Introdução}

O termo desnutrição tem sido usado para definir um estado de carência de proteínas, energia ou outros nutrientes necessários para o crescimento, desenvolvimento, manutenção e recuperação das funções do organismo. Seu reconhecimento pode ser feito através de sinais e sintomas como: cabelos fracos e ralos, sem pigmentação e fáceis de arrancar; edema de face, mãos e pés; seborreia nasolabial, alterações psicomotoras, da marcha, fraqueza e formigamento das extremidades, entre outros ${ }^{1}$.

Em uma pessoa com deficiência nutricional ou desnutrição - com baixos níveis séricos de glicose, eletrólitos e vitaminas, entre elas, a tiamina - quando recebem carboidratos, especialmente a glicose por via endovenosa, podem desenvolver, em até 72 horas do início da reintrodução de nutrientes, um quadro de Síndrome de Realimentação ${ }^{2}$. Nessa situação verifica-se aumento da insulina ${ }^{3}$, o que inibe a proteólise, impedindo a sua utilização para produção de albumina, agravando os níveis séricos dos eletrólitos que já estavam alterados, principalmente o fósforo ${ }^{4,5}$.

Diante desses aspectos, destaca-se a Síndrome da Realimentação (SR) que, ainda hoje, é pouco conhecida pelos profissionais de saúde. Tal quadro pode ocorrer em qualquer idade e em qualquer indivíduo com depleção nutricional ou desnutrição, quando submetido a um jejum prolongado ou mesmo privação alimentar por inapetência, anorexia, greve de fome, má absorção alimentar, condição de subalimentação, entre outras situações ${ }^{2}$. Este quadro foi observado, inicialmente, entre os sobreviventes da Segunda Guerra Mundial e intrigou os pesquisadores, o que os levou a estudar este fenômeno que foi, posteriormente, denominado de Síndrome da Realimentação $0^{6,7}$.

Na SR, a mobilização energética resulta em perda de massa muscular e perda intracelular dos eletrólitos, que em pessoas desnutridas, podem estar esgotadas². A hipofosfatemia (menor que um $\mathrm{mmol} / \mathrm{L}$ ) surge em média próximo ao segundo dia da realimentação ${ }^{3}$, sendo um sinal de alerta precoce para SR. A sintomatologia depende diretamente do eletrólito mais deficiente e, no geral, quando estes pacientes evoluem ao óbito, não são relacionados à SR, mas, a outros motivos ${ }^{6}$. Por isso, devido ao pouco conhecimento sobre a SR, crianças, adultos e idosos em depleção nutricional situação em que se observa o principal risco para SR - não são identificados e, quando desenvolvem a SR, não são tratados adequadamente ${ }^{2}$.

Tais aspectos são importantes, assim como identificar sinais e sintomas sugestivos de desnutrição, o que pode levar à prevenção do desenvolvimento da SR como: IMC abaixo de $18,5 \mathrm{~kg} / \mathrm{m}^{6}$, perda de peso involuntária maior que $10 \%$ do peso habitual, num período de três a seis meses e diminuição na circunferência da panturrilha são indicativos de depleção nutricional, além dos baixos níveis de proteína $C$ reativa, interleucina-6, hemoglobina (abaixo de $12 \mathrm{~g} / \mathrm{dL}$ ) e albumina (menor que 3,2 g/dL) ${ }^{1}$.

O Quadro 1, a seguir, apresenta os critérios que fundamentam o quadro de risco para desenvolver a SR:

Quadro 1. Critérios para identificação de risco para desenvolver a SR.

\begin{tabular}{lcc}
\multicolumn{1}{c}{ Alterações } & $\begin{array}{c}\text { Condição A } \\
\text { (um ou mais critérios) }\end{array}$ & $\begin{array}{c}\text { Condição B } \\
\text { (dois ou mais critérios) }\end{array}$ \\
IMC & $<16\left(\mathrm{~kg} / \mathrm{m}^{2}\right)$ & $<18\left(\mathrm{~kg} / \mathrm{m}^{2}\right)$ \\
\hline $\begin{array}{l}\text { Perda de peso involuntária em } 3 \\
\text { a } 6 \text { meses anteriores }\end{array}$ & $>15 \%$ & $>10 \%$ \\
\hline $\begin{array}{l}\text { Número de dias com restrição de } \\
\text { nutrientes }\end{array}$ & $>10$ & $>5$ \\
\hline Outros fatores & $\begin{array}{c}\text { Diminuição dos níveis } \\
\text { sanguíneos de fósforo, potássio e } \\
\text { magnésio. }\end{array}$ & $\begin{array}{c}\text { Histórico de abuso de álcool ou } \\
\text { outras drogas. }\end{array}$ \\
\hline
\end{tabular}

Fonte: Caldas e Alves ${ }^{6}$. 
Os sinais e sintomas da SR também estão diretamente relacionados às alterações dos eletrólitos alterados no sangue ${ }^{8}$. Sintomas como agitação psicomotora, rebaixamento do nível de consciência, convulsão, edema, insuficiência respiratória, obstipação ou diarreia, contrações musculares involuntárias, arritmia cardíaca, falência cardíaca e morte são sinais importantes para levantar a hipótese de SR . O Quadro 2, a seguir, apresenta os parâmetros de risco para o diagnóstico de SR.

No Quadro 3, a seguir, apresenta-se a conduta terapêutica mais adequada para os pacientes em risco ou que já desenvolveram a SR.

Quadro 2. Parâmetros de risco para o diagnóstico da SR.

\begin{tabular}{lc}
\multicolumn{1}{c}{ Característica bioquímica/clínica } & Parâmetro de risco para SR \\
\hline Potássio sérico & abaixo de $2,5 \mathrm{mmol} / \mathrm{L}$ \\
\hline Fosforo sérico & abaixo de $0,32 \mathrm{mmol} / \mathrm{L}$ \\
\hline Magnésio & menor que $0,5 \mathrm{mmo} / \mathrm{L}$ \\
\hline Edema periférico & presente \\
\hline Líquidos no corpo & aumentado \\
\hline $\begin{array}{l}\text { Disfunção de órgãos e sistemas (especialmente } \\
\text { respiratórios) }\end{array}$ & presente \\
\hline
\end{tabular}

Fonte: Caldas e Alves'.

Quadro 3. Condutas terapêuticas necessárias para pacientes em risco de SR: metas para início e evolução nutricional.

\begin{tabular}{lcc} 
Metas diárias & IMC $<\mathbf{1 4} \mathbf{~ k g} / \mathbf{m}^{2}$ ou jejum $>\mathbf{1 5}$ dias & IMC $<\mathbf{1 8 ~} \mathbf{~ k g} / \mathbf{m}^{2}$ \\
\hline Meta calórica & $\begin{array}{c}5 \mathrm{kcal} / \mathrm{kg} / \text { dia } \\
\text { aumentando lentamente: } \\
5 \mathrm{kcal} / \mathrm{kg} / \text { dia em até } 7 \text { dias }\end{array}$ & $\begin{array}{c}10 \mathrm{kcal} / \mathrm{kg} / \text { dia } \\
\text { aumentando lentamente: } \\
5 \mathrm{kcal} / \mathrm{kg} / \mathrm{dia} \text { em até } 7 \mathrm{dias}\end{array}$ \\
\hline Meta proteica & $1,2 \mathrm{a} 1,5 \mathrm{~g} / \mathrm{kg} /$ dia & $1,2 \mathrm{a} 1,5 \mathrm{~g} / \mathrm{kg} / \mathrm{dia}$ \\
\hline Meta de glicose & $100 \mathrm{a} 200 \mathrm{~g} /$ dia & $100 \mathrm{a} 200 \mathrm{~g} /$ dia \\
\hline Meta de lipídio & $\begin{array}{c}20 \text { a } 30 \% \text { das calorias } \\
\text { não proteicas }\end{array}$ & $\begin{array}{c}20 \text { a } 30 \% \text { das calorias } \\
\text { não proteicas }\end{array}$ \\
\hline
\end{tabular}

Fonte: $\mathrm{NICE}^{9}$.

A meta calórica nos dois primeiros dias, após a identificação do paciente em risco para desenvolver SR, deve ser de 50\% das necessidades calóricas diárias. A dosagem dos eletrólitos e a monitorização cardíaca se faz necessária, antes da reintrodução de nutrientes e após, no mínimo por quatro dias, devido ao risco de arritmia cardíaca, que é a principal causa de óbito na SR 6 .

Diante dessas considerações, observa-se que é fundamental que os profissionais de saúde, principalmente os médicos que trabalham em contexto hospitalar, sejam capazes de identificar os sinais e sintomas que podem levar ao desenvolvimento da SR. Ao identificar esse risco, a Equipe Multidisciplinar de Terapia Nutricional (EMTN) hospitalar deve ser acionada a fim de iniciar um planejamento nutricional adequado.

Portanto, o presente artigo tem como objetivo caracterizar os conhecimentos e práticas de uma equipe multiprofissional de saúde de alta complexidade quanto aos quadros de desnutrição e a SR. Além disso, a partir das informações colhidas na pesquisa, pretende-se embasar a construção de um Produto Técnico em Educação (PTE) na forma de um protocolo de avaliação multiprofissional para subsidiar um processo de Educação Permanente (EP) sobre este quadro complexo e fatal. 


\section{Método}

Foi realizada uma pesquisa ${ }^{10}$ de Mestrado no Programa de Pós-graduação Ensino em Ciências da Saúde - Modalidade Profissional da Universidade Federal de São Paulo (UNIFESP), baseada na metodologia qualitativa e usando a técnica do grupo focal com profissionais de saúde que atuam em uma unidade de terapia intensiva de um hospital geral municipal na cidade de Santos - na região da Baixada Santista-São Paulo - Brasil. A coleta de dados ocorreu entre os meses de abril a dezembro de 2017.

A estratégia do grupo focal ${ }^{11}$, também conhecida como entrevista aberta grupal, é uma técnica qualitativa, cujo objetivo consiste em captar percepções, angústias, ansiedades e ideias, fazendo emergir uma multiplicidade de pontos de vista e processos emocionais, pelo próprio contexto de interação criado. Na pesquisa realizada, tal estratégia possibilitou uma condução menos diretiva por parte dos pesquisadores e maior interação entre os membros, na qual se privilegiaria a expressão grupal, visando a apreensão do estado atual dos conhecimentos a partir do cotidiano de trabalho dos participantes sobre o assunto e permitir uma troca de experiências o que, por si só, pode levar a uma reflexão e sensibilização sobre o risco da SR em pacientes internados.

Foram realizados dois grupos focais ${ }^{11}$ com agendamento antecipadamente combinado, durante o horário de trabalho e em sala reservada no hospital que continha uma mesa grande no qual o grupo se acomodou em torno, cadeiras, ar condicionado e privacidade para a realização da atividade.

Os participantes dos grupos foram profissionais de saúde de um hospital geral do município, que realizam atendimento direto à pacientes críticos da UTI (Unidade de Terapia Intensiva). Os participantes foram selecionados pela função profissional exercida, devido aos seus diferentes olhares, experiências e autonomia profissional. Nos dois grupos focais realizados foi mantida a escolha das mesmas profissões, com o propósito de manter um resultado homogêneo entre os grupos. No primeiro grupo foram sete participantes e, no segundo grupo, foram seis participantes.

Como critérios de inclusão, cabe destacar que todos participantes são servidores públicos que atuam diretamente na assistência aos pacientes internados na UTI e foram convidados a participar da pesquisa considerando que os mesmos podem contribuir para a identificação de pessoas em risco para SR no serviço de saúde, sendo eles: profissionais das áreas de Medicina, Enfermagem, Nutrição, Fisioterapia, Psicologia e ajudantes de cozinha. Como critério de exclusão, foram excluídos os profissionais que não fossem formalmente vinculados ao serviço público, que não trabalhavam diretamente prestando assistência aos pacientes na UTI e que não concordaram em assinar formalmente o Termo de Consentimento Livre e Esclarecido (TCLE).

Cada grupo focal teve a duração de aproximadamente uma hora e as temáticas abordadas nos grupos focais foram propostas a partir da literatura científica sobre o assunto e a experiência profissional da pesquisadora, sendo elas: conhecimentos sobre desnutrição e síndrome da realimentação em pacientes adultos internados. Os grupos foram moderados pela pesquisadora e também contaram com a participação de uma observadora que tinha a função, além de observação e auxílio no registro da atividade, a observadora também contribuía em conduzir situações possíveis de interrupções ou outras necessidades de ordem logística.

Os grupos focais foram realizados de modo semiestruturado, com o uso de um roteiro temático de questões norteadoras elaborado pela pesquisadora com o intuito de fomentar discussões e trocas de experiências. Além disto, utilizou-se de uma atividade estruturada, na qual se apresentaram aos participantes do grupo focal uma série de imagens disponíveis na internet e selecionadas pela pesquisadora. Tais imagens eram de adultos desnutridos e potencialmente em risco para desenvolver SR. As imagens foram cuidadosamente tarjadas para evitar o reconhecimento das pessoas e serviram de base para a discussão sobre o risco de desenvolvimento da SR. 
Os discursos dos participantes dos grupos foram gravados e transcritos fielmente pela pesquisadora e os mesmos forma analisados e categorizados pelo método de Análise de Conteúdo temática ${ }^{12}$. Todos os participantes dos serviços foram convidados a participar da pesquisa e os que aceitaram assinaram um TCLE, conforme a Resolução 466/12 do CNS. A pesquisa foi aprovada pelo Comitê da Unifesp sob $\mathrm{n}^{\circ}$ CAAE 64690117.2.0000.5505 e número do parecer final 1.976.053.

\section{Resultados}

Participaram dos grupos focais um total de 13 pessoas, sendo sete no primeiro e seis no segundo grupo. O tempo médio de experiência profissional dos participantes foi de 13,3 anos (mínimo de quadro e máximo de 29 anos). Quatro participantes eram integrantes da Equipe Multiprofissional de Terapia Nutricional (EMTN).

Como foi observado nos discursos dos participantes durante as discussões dos grupos focais, há um desconhecimento significativo sobre a SR, o que, de certa forma era um dado esperado. Porém, chamou a atenção o fato de que, diante das imagens, houve uma dificuldade de reconhecer adultos em desnutrição, mesmo entre os médicos. Essa dificuldade se apresentou independentemente do tempo de experiência e da formação profissional.

Cabe realçar que a maioria dos participantes reconheceu que as imagens apresentadas nas discussões dos grupos focais são muito próximas à experiência que os profissionais têm com pacientes internados no hospital e na UTI. Diante das imagens propostas, pessoas desnutridas crônicas, mista, ou do tipo Khwaskiokor, com peso normal, ou sobrepeso para a idade, devido ao edema, não foram identificadas como portadoras de depleção nutricional e, como consequência disso, não são identificadas como em potencial risco para desenvolver a SR.

As discussões dos grupos focais giraram em torno das experiências prévias dos participantes em suas atividades profissionais e que, no geral, entendiam que os casos de desnutrição estariam associados apenas à infância. Referiram que, entre adultos, os quadros de desnutrição estão associados com algumas doenças como câncer ou idosos gravemente adoecidos.

Os profissionais médicos que participaram da pesquisa referiram que a desnutrição e a perda de massa muscular foram indicativas de mau prognóstico, e que, na prescrição médica - que é uma ferramenta de comunicação do médico com os demais profissionais de saúde - reconheceram que há carência de domínio na prescrição dietética, por provável lacuna na formação acadêmica na temática sobre nutrição.

Outro ponto digno de atenção foi o fato de que nas narrativas dos profissionais participantes houve relatos de preocupação e angústia em lidar com indivíduos internados que se apresentam com magreza extrema. Relataram que quando o paciente apresenta tal fragilidade física, entendem que isso pode ter relação com o fenômeno da "fome", típico de condições de extrema pobreza, o que costuma gerar muita comoção emocional e uma tendência a tentar alimentá-lo com muitas calorias e de forma rápida, o que, afinal, pode levar ao risco para desenvolvimento da SR.

\section{Discussão}

Considerando que a desnutrição hospitalar é reconhecida como um problema relevante, pois se estima que 20 a $50 \%$ dos adultos internados apresentam desnutrição ${ }^{13}$, tal dado nos leva a considerar grave potencialização do risco de desenvolvimento da SR, especialmente diante de manejo inadequado e pouca resolutividade ${ }^{6-14}$.

Nas UTIs observa-se a maior prevalência de desnutrição com perda de massa muscular ${ }^{15}$, o que nos faz entender que, de um ponto de vista lógico, trata-se de um local de maior probabilidade de que um paciente desenvolva SR, principalmente 
diante do fácil acesso de infusões intravenosas de glicose com rápida absorção, comparada a via oral ${ }^{13}$.

Diante das informações da literatura sobre o assunto - ainda que escassas ou pouco divulgadas - e dos achados da pesquisa realizada pelos autores deste presente artigo, ressalta-se que a identificação de pacientes em risco de SR é uma questão relevante e necessária, principalmente no contexto hospitalar. Os dados coletados indicam que há um desconhecimento generalizado sobre a SR e que, além disso, muitos participantes relataram uma tendência em intervir com aumento do aporte calórico e de modo abrupto em casos de pacientes desnutridos, o que leva ao risco aumentado para esta síndrome. Portanto, essas informações permitem considerar que as condutas relatadas pelos profissionais participantes do estudo, no caso de quadros de desnutrição em adultos, sugerem desconhecimento quanto ao diagnóstico e terapêuticas adequadas, o que pode interferir no prognóstico em questão.

Outro achado da pesquisa foi identificar que há uma dificuldade de reconhecer adultos em desnutrição, mesmo entre os médicos, o que nos leva a refletir sobre uma lacuna na formação acadêmica na área da saúde sobre essa temática, assim como outros autores referem em suas pesquisas ${ }^{16}$.

Embora existam poucos trabalhos que tratem esse assunto a partir de um olhar de Saúde Pública, um estudo observou essa mesma dificuldade de reconhecer um adulto em desnutrição, pois somente $1,2 \%$ dos pacientes com SR foram diagnosticados ${ }^{6}$. Outro estudo ${ }^{14}$ explicita que a SR é um quadro nosográfico esquecido e potencialmente fatal. Por sua vez, outro estudo ${ }^{17}$ refere que a SR foi descrita a mais de 70 anos, porém continua pouco estudada e com muitas lacunas na definição padronizada e tratamento recomendado. Cabe ressaltar que a presente pesquisa realizada corrobora com as reflexões desses autores que relataram uma desvalorização ou desconhecimento desse problema de saúde, mesmo com toda evolução tecnológica no campo de saúde.

\section{Conclusão}

A pesquisa realizada com o instrumento dos grupos focais se mostrou bastante potente, com grande potencial interativo, compartilhamento de experiências e vivências de forma espontânea e produtiva.

Embora alguns poucos participantes tivessem algum conhecimento teórico sobre desnutrição e SR, observou-se dificuldade generalizada para o identificar imagens de pacientes em risco para desenvolver a SR, além de desconexão entre os conhecimentos teóricos e sua prática profissional cotidiana.

Além dos dados obtidos nessa pesquisa, os autores se basearam nas discussões atualizadas sobre o "Nutritional Risk Screening 2002"18, para - seguindo a proposta de um Mestrado Profissional - criar um PTE na forma de um protocolo de avaliação multiprofissional intitulado "Protocolo resumido interdisciplinar de avaliação e conduta para risco de Síndrome da Realimentação SR"19. Este PTE foi apresentado na Figura 1 (parte da frente do documento construído) e Figura 2 (parte de trás do documento construído).

Pretende-se que os serviços de saúde onde foi realizada a pesquisa utilizem deste protocolo de modo que o uso deste instrumento para avaliação do risco nutricional em adultos, principalmente nos casos de jejum por mais de 48 horas, seja implantado de modo efetivo e continuado.

Diante dos achados desta pesquisa, as autoras entendem que ações contínuas de formação nos serviços de saúde mostram-se fundamentais para a construção de uma prática de cuidado em saúde realmente capaz de identificar indivíduos em risco para desenvolver SR e acionar condutas profiláticas adequadas para evitar, inclusive, o risco de óbito. 


\begin{tabular}{|c|c|c|c|}
\hline \multicolumn{4}{|c|}{ 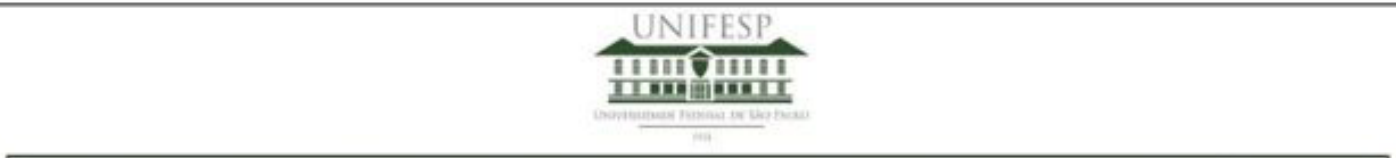 } \\
\hline \multicolumn{4}{|c|}{$\begin{array}{l}\text { PROTOCOLO RESUMIDO INTERDISCIPLINAR DE AVALIAÇÃO E } \\
\text { CONDUTA PARA RISCO DE SÍNDROME DA REALIMENTAÇÃO (SR) }\end{array}$} \\
\hline \multicolumn{4}{|c|}{$\begin{array}{l}\text { Citação: CARVALHO MS, ZIHLMANN KF. Protocolo resumido interdisciplinar de avaliação e conduta } \\
\text { para risco de Síndrome da Realimentação (SR). [Produto Técnico Educacional]. Santos: Universidade } \\
\text { Federal de São Paulo - Campus Baixada Santista - UNIFESP-BS; } 2018 . \\
\text { Todos os direitos deste protocolo são reservados. Este documento não pode ser reproduzido, todo ou em parte, ou cedido, incluindo } \\
\text { fotocópias, nem armażenado em sistema informático, sem a autorização escrita prévia dos autores. Os interessados poderão fażer } \\
\text { contato com os autores, com intuito de aplicação em atividades clínicas e de pesquisa. }\end{array}$} \\
\hline DEFINIÇÃO & \multicolumn{3}{|c|}{$\begin{array}{l}\text { A SR é o conjunto de alterações clínicas e eletrolíticas que se inicia após } \\
\text { reintroduzir alimentos (principalmente glicose) por via endovenosa, } \\
\text { enteral ou oral (carboidratos) em uma pessoa em privação de alimentos ou } \\
\text { desnutrição, que pode levar o paciente ao óbito em poucos dias. }\end{array}$} \\
\hline $\begin{array}{l}\text { Sinais de alerta } \\
\text { para identificação }\end{array}$ & \multicolumn{3}{|c|}{$\begin{array}{l}\text { Paciente com sinais de desnutrição (mesmo em pacientes acima do } \\
\text { peso) e/ou privação alimentar por mais de } 15 \text { dias; }\end{array}$} \\
\hline $\begin{array}{l}\text { dos pacientes em } \\
\text { risco de SR } \\
\text { (comunicar à EMTN) }\end{array}$ & \multirow{2}{*}{\multicolumn{2}{|c|}{$\begin{array}{l}>\text { Arritmia cardíaca; } \\
>\text { Desconforto respiratório; } \\
>\text { Hipo ou hiperglicemia; }\end{array}$}} & $\begin{array}{l}>\text { Eletrólitos baixos } \\
\text { (principalmente fósforo); } \\
>\text { Edema ou anasarca. }\end{array}$ \\
\hline \multicolumn{2}{|l|}{ Fonte: Caldas (2015) } & & \\
\hline \multicolumn{4}{|c|}{ Quadro 1 - Critérios para identificar indivíduos em risco para desenvolver SR } \\
\hline \multicolumn{2}{|l|}{ Alterações } & $\begin{array}{c}\text { Condição A } \\
\text { (um ou mais critérios) }\end{array}$ & $\begin{array}{l}\text { Condição B } \\
\text { (dois ou mais critérios) }\end{array}$ \\
\hline IMC $=$ Peso $(\mathrm{kg}) /$ altu & $\left(\mathrm{m}^{2}\right)$ & $<16\left(\mathrm{~kg} / \mathrm{m}^{2}\right)$ & $<18\left(\mathrm{~kg} / \mathrm{m}^{2}\right)$ \\
\hline \multicolumn{4}{|l|}{ Condições } \\
\hline \multicolumn{2}{|c|}{$\begin{array}{l}\text { Perda de peso involuntária entre } \\
3 \text { a } 6 \text { meses anteriores }\end{array}$} & $>15 \%$ & $>10 \%$ \\
\hline \multicolumn{2}{|c|}{$\begin{array}{l}\text { Número de dias restrição de } \\
\text { nutrientes (alimentos) }\end{array}$} & $>10$ & $>5$ \\
\hline \multicolumn{2}{|l|}{ Outros fatores } & $\begin{array}{l}\text { diminuição dos níveis } \\
\text { sanguíneos de potássio, } \\
\text { fósforo, magnésio }\end{array}$ & $\begin{array}{l}\text { história de abuso de álcool e } \\
\text { outras drogas }\end{array}$ \\
\hline \multicolumn{4}{|l|}{ Fonte: Rio (2013) } \\
\hline \multicolumn{4}{|c|}{ Quadro 2 - CONDUTA: Metas para início e evolução nutricional* } \\
\hline Metas diárias & \multicolumn{2}{|c|}{ IMC $<14 \mathrm{~kg} / \mathrm{m}^{2}$ ou jejum $>15$ dias } & IMC $>18 \mathrm{~kg} / \mathrm{m}^{2}$ \\
\hline $\begin{array}{l}\text { Meta calórica } \\
\text { (nos primeiros } 2 \text { dias: } \\
50 \% \text { das } \mathrm{NC} \text { ) }\end{array}$ & \multicolumn{2}{|c|}{$\begin{array}{l}5 \mathrm{kcal} / \mathrm{kg} / \mathrm{dia} \\
\text { aumentando lentamente: } \\
5 \mathrm{kcal} / \mathrm{kg} / \mathrm{dia} \text { em até } 7 \text { dias }\end{array}$} & $\begin{array}{l}10 \mathrm{kcal} / \mathrm{kg} / \mathrm{dia} \\
\text { aumentando lentamente: } \\
5 \mathrm{kcal} / \mathrm{kg} / \text { dia em até } 7 \text { dias }\end{array}$ \\
\hline Meta proteica & \multicolumn{2}{|c|}{1,2 a $1,5 \mathrm{~g} / \mathrm{kg} / \mathrm{dia}$} & 1,2 a $1,5 \mathrm{~g} / \mathrm{kg} / \mathrm{dia}$ \\
\hline Meta de glicose & \multicolumn{2}{|c|}{100 a 200 g/dia } & 100 a $200 \mathrm{~g} / \mathrm{dia}$ \\
\hline Meta de lipídio & \multicolumn{2}{|c|}{20 a $30 \%$ das calorias não proteicas } & $\begin{array}{l}20 \text { a } 30 \% \text { das calorias não } \\
\text { proteicas }\end{array}$ \\
\hline $\begin{array}{l}\text { Eletrólitos } \\
\text { (antes e após } \\
\text { realimentaçấo) }\end{array}$ & \multicolumn{2}{|c|}{ Monitorar por 4 dias } & Monitorar por 4 dias \\
\hline \multicolumn{4}{|l|}{ Fonte: NICE (2006) } \\
\hline
\end{tabular}

Figura 1. Parte da frente do Protocolo resumido interdisciplinar de avaliação e conduta para risco de Síndrome da Realimentação. 


\section{MEDIDAS PREVENTIVAS E TERAPEUTICAS}

\begin{tabular}{|l|l|}
\hline \multicolumn{1}{|l}{ MEDIDAS PREVENTIVAS E TERAPEUTICAS } \\
\hline & $\begin{array}{l}\text { 1) Identificar os critérios de risco para SR; } \\
\text { 2) Controle de peso diário de todos pacientes, se possível; } \\
\text { 3) Aquecimento do corpo, principalmente das extremidades, evitar } \\
\text { lesões por pressão e esforço físico (ativo). }\end{array}$ \\
\hline Médico & $\begin{array}{l}\text { 1) Solicitar exames de: glicose, sódio, potássio, magnésio, fósforo, } \\
\text { cálcio, antes de iniciar a alimentação (oral, enteral ou glicose } \\
\text { endovenosa); } \\
\text { (Não realizar infusão de glicose endovenosa em paciente } \\
\text { identificado de risco para desenvolver SR) } \\
\text { 2) Hidratação adequada e cautelosa, devido a tendência para retenção } \\
\text { de sódio e água destes pacientes; } \\
\text { (Não é necessário normalizar as alterações eletrolíticas antes do } \\
\text { início da terapia nutricional (TN); } \\
\text { 3) Em caso de alterações graves, como fósforo < 1mg/dL, magnésio } \\
\text { <1mg/ dL, potássio: corrigir as alterações antes de iniciar a TN; } \\
\text { (Se níveis séricos baixos, porém superiores aos descritos acima, } \\
\text { iniciar correção concomitante ao início do suporte nutricional;) } \\
\text { 4) Suplementação venosa de vitaminas e oligoelementos: iniciar } \\
\text { antes de começar a TN e manter até atingir o 7º dia. }\end{array}$ \\
\hline Fisioterapeuta
\end{tabular}

Figura 2. Parte de trás do Protocolo resumido interdisciplinar de avaliação e conduta para risco de Síndrome da Realimentação.

Por fim, a pesquisa evidenciou que a SR pode ser considerada um quadro negligenciado em Saúde Pública, o que leva à necessidade de aprofundar conhecimentos dessa temática nas graduações da área da saúde e, além disso, fomentar ações de Educação Permanente nos serviços de saúde de modo a abordar adequadamente as necessidades de cuidado específicas desses pacientes.

\section{Referências}

1. Mahan LK. Krause: alimentos, nutrição e dietoterapia. 13. ed. Rio de Janeiro: Elsevier; 2012. 


\section{ijhe}

2. Viana LA, Burgos MGPA, Silva RA. Qual é a importância clínica e nutricional da síndrome de realimentação? Arq Bras Cir Dig. 2012;25(1):56-9. http://dx.doi.org/10.1590/S0102-67202012000100013.

3. Caruso L, Sousa AB. Manual da equipe multidisciplinar de terapia nutricional (EMTN) - Hospital Universitário da Universidade de São Paulo [Internet]. São Carlos: Cubo; 2014 [citado em 2021 Abr 20]. Disponível em: http://www.hu.usp. br/manual-da-equipe-multidisciplinar-de-terapia-nutricional

4. Olthof LE, Koekkoek WACK, van Setten C, Kars JCN, van Blokland D, Van Zanten ARH. Impact of caloric intake in critically ill patients with, and without, refeeding syndrome: a retrospective study. Clin Nutr. 2018;37(5):1609-17. http:// dx.doi.org/10.1016/j.clnu.2017.08.001. PMid:28866139.

5. Castro MA, Vázquez Martínez C. The refeeding syndrome. Importance of phosphorus. Med Clin (Barc). 2018;150(12):4728. http://dx.doi.org/10.1016/j.medcli.2017.12.008. PMid:29448987.

6. Caldas AC, Alves JTM. Síndrome de realimentação em pacientes hospitalizados: série de casos e revisão da literatura. Int J Neurol. 2015;8(2):22-9. http://dx.doi.org/10.1055/s-0040-1705068.

7. Reber E, Friedli N, Vasiloglou MF, Schuetz P, Stanga Z. Management of refeeding syndrome in medical inpatients. J Clin Med. 2019;8(12):2202. http://dx.doi.org/10.3390/jcm8122202. PMid:31847205.

8. Nealis TB, Buchman A. Enteral and parenteral nutrition [Internet]. ACP Medicine; 2011. 19 p. [citado em 2021 Abr 20 ]. Disponível em: http://www.medicinanet.com.br/conteudos/acp-medicine/5644/nutricao_enteral_e_parenteral_\%E2\%80\%93_ thomas_b_nealis_alan_buchman.htm

9. National Institute for Health and Care Excellence (NICE). Suporte nutricional para adultos: suporte à nutrição oral, alimentação por tubo enteral e nutrição parenteral. Diretriz CG32 [Internet]. Londres; 2020 [citado em 2020 Out 25]. Disponível em: https://www.nice.org.uk/guidance/cg32/chapter/1-Guidance\#more-information

10. Carvalho MS. Síndrome da realimentação: um olhar interdisciplinar sobre um quadro negligenciado e fatal [dissertação]. Santos: Universidade Federal de São Paulo; 2018.

11. Gatti BA. Grupo focal na pesquisa em ciências sociais e humanas. Brasília: Liber Livro; 2005.

12. Bardin L. Análise de conteúdo. São Paulo: Edições 70; 2011

13. Toledo DO, Piovacari SMF, Horie LM, et al. Campanha "Diga não à desnutrição": 11 passos importantes para combater a desnutrição hospitalaro. BRASPEN J [Internet]. 2018 [citado em 2020 Out 25];33(1):86-100. Disponível em: http://arquivos. braspen.org/journal/jan-fev-mar-2018/15-Campanha-diga-nao-aadesnutricao.pdf

14. Aissaoui Y, Hammi S, Tagajdid R, Chkoura K, Boughalem M. Refeeding Syndrome: a forgotten and potentially lethal entity. Med Sante Trop. 2016;26(2):213-5. http://dx.doi.org/10.1684/mst.2015.0513. PMid:27412982.

15. Puthucheary ZA, Rawal J, McPhail M, et al. Acute skeletal muscle wasting in critical illness. JAMA. 2013;310(15):1591600. http://dx.doi.org/10.1001/jama.2013.278481. PMid:24108501.

16. Janssen G, Pourhassan M, Lenzen-Großimlinghaus R, et al. The Refeeding Syndrome revisited: you can only diagnose what you know. Eur J Clin Nutr. 2019;73(11):1458-63. http://dx.doi.org/10.1038/s41430-019-0441-x. PMid:31127188.

17. Friedli N, Stanga Z, Sobotka L, et al. Revisiting the refeeding syndrome: results of a systematic review. Nutrition. 2017;35:15160. http://dx.doi.org/10.1016/j.nut.2016.05.016. PMid:28087222.

18. Barbosa AAO, Vicentini AP, Langa FR. Comparação dos critérios da NRS-2002 com o risco nutricional em pacientes hospitalizados. Cienc Saúde Colet. 2019;24(9):3325-34. http://dx.doi.org/10.1590/1413-81232018249.25042017.

19. Carvalho MS, Zihlmann KF. Protocolo resumido interdisciplinar de avaliação e conduta para risco de Síndrome da Realimentação (SR) [produto técnico educacional]. Santos: Universidade Federal de São Paulo; 2018.

\section{Contribuição dos autores}

Marcia Souza Carvalho concepção e execução do trabalho, coleta e análise de dados, elaboração da versão final do artigo. Karina Franco Zihlmann orientação e concepção do trabalho, análise de dados, elaboração da versão final do artigo. 